\title{
Efficacy of photobiomodulation therapy in with hip osteoarthritis
}

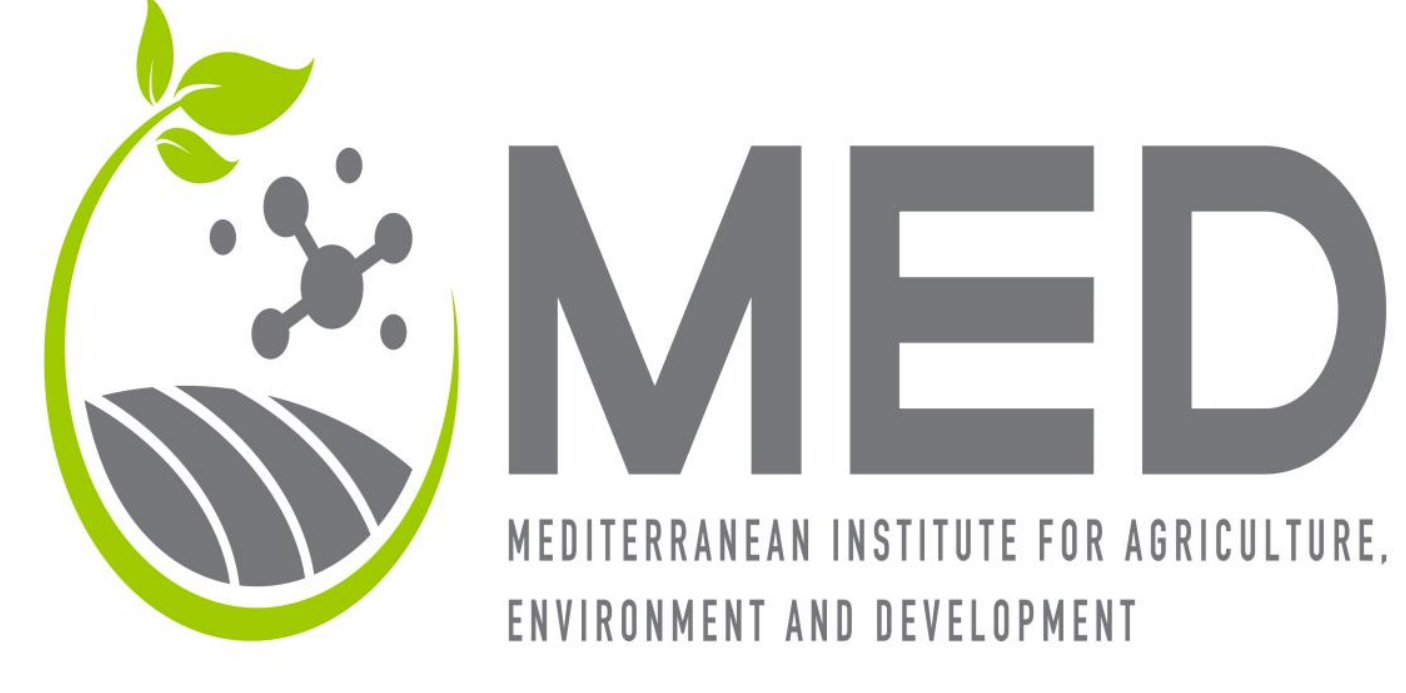

\section{Alves J.1,2,*; Jorge P. '; Santos A. '; Carreira, L. Miguel2,3}

'Guarda Nacional Republicana; ' MED - Mediterranean Institute for Agriculture, Environment and Development, Instituto de Investigação e Formação Avançada, Universidade de Évora. ${ }^{3}$ Interdisciplinary Centre for Research in Animal Health (CIISA) - University of Lisbon, (FMV/ULisboa) - Portugal.

${ }^{4}$ Anjos of Assis Veterinary Medicine Centre; *alves.jca@gnr.pt

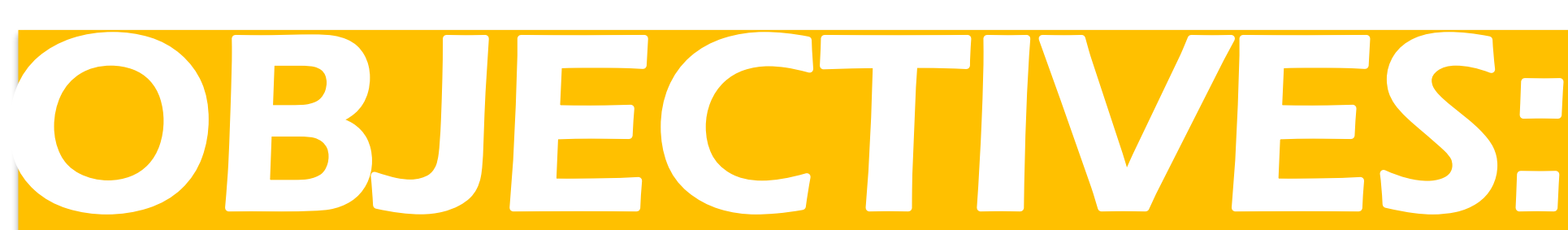

To describe the effect of photobiomodulation therapy in dogs with hip osteoarthritis (OA).

\section{MATERIALS AND NETHODSE}

Forty joints were assigned to a control group (CG, $n=20$ ) or treatment group (PBMT, $n=20$ ). CG received a 21-day course of meloxicam, and PBMT received treatment with a Class IV therapeutic laser, divided into three sessions on week one, two sessions on week two, and one session on week three.

Joint range of motion, thigh girth, the Canine Brief Pain Inventory (CBPI, divided into pain interference score - PIS and Pain Severity Score - PSS), Liverpool Osteoarthritis in Dogs (LOAD) and Canine Orthopedic Index (COI, divided into four dimensions: function, gait, stiffness, and quality of life - OOL) were evaluated before treatment, $+8,+15,+30,+60$ and +90 days after initial treatment.

Results were analyzed with repeated measures ANOVA or Wilcoxon signed ranks test. Kaplan-Meier estimators were conducted and compared with the Breslow test , $p<0.05$.

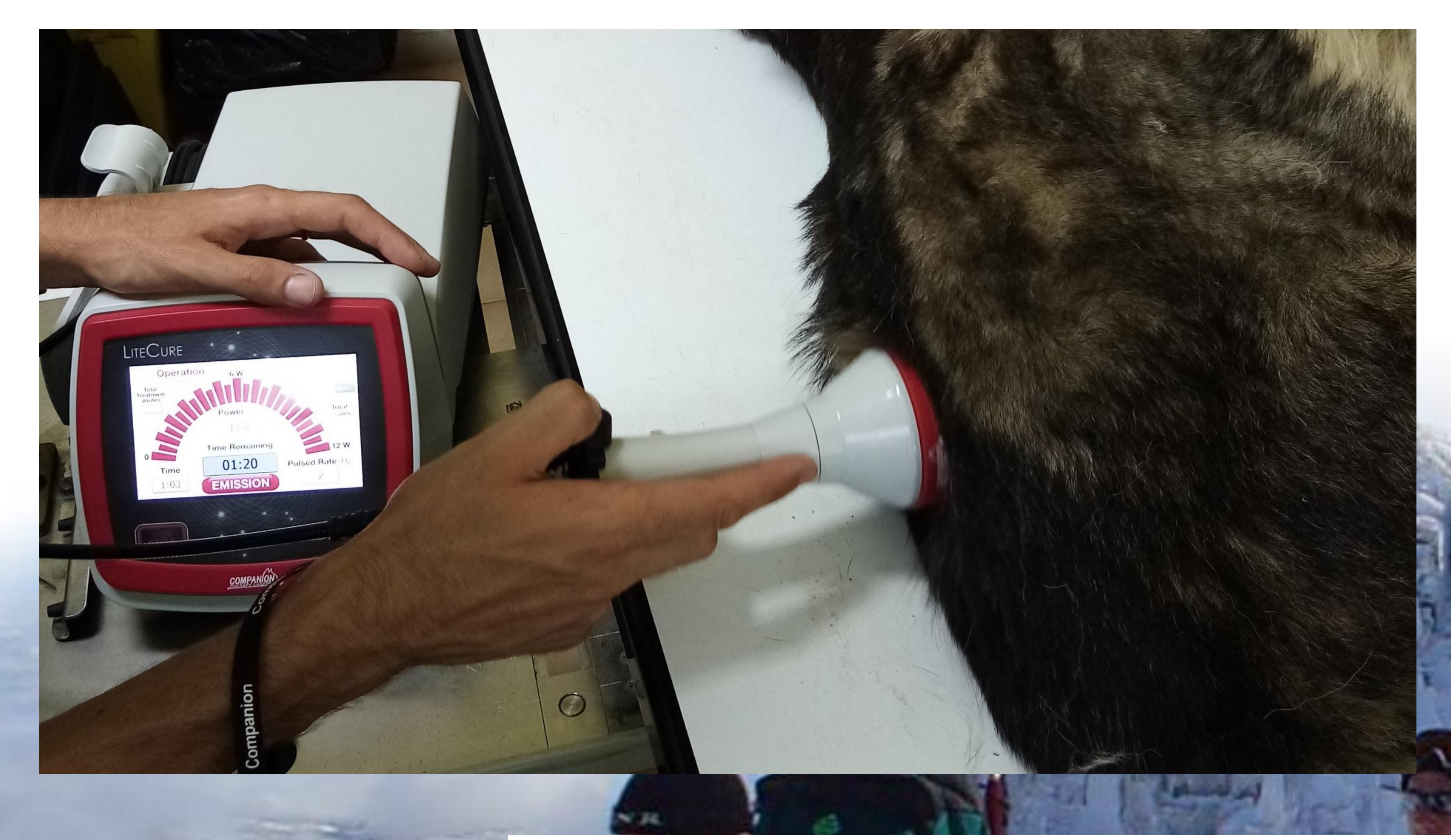

No significant differences were observed at the initial evaluation. Better results were observed in PBMT at $+8 d$ $(p=0.01$ for PSS, $p=0.04$ for function and COI) $+15 d(p=0.01$ for PSS and function, $p=0.02$ for PIS and function, $p=0.03$ for COI and $p=0.04$ for LOAD) and $+30 d(p=0.01$ for function and gait, $p=0.02$ for $C O I$ and $p=0.04$ for PIS, PSS and LOAD). The joint range of motion improved in PBMT from $+15 d$ to +90 d. Kaplan-Meier estimators showed better results in the PBMT group.
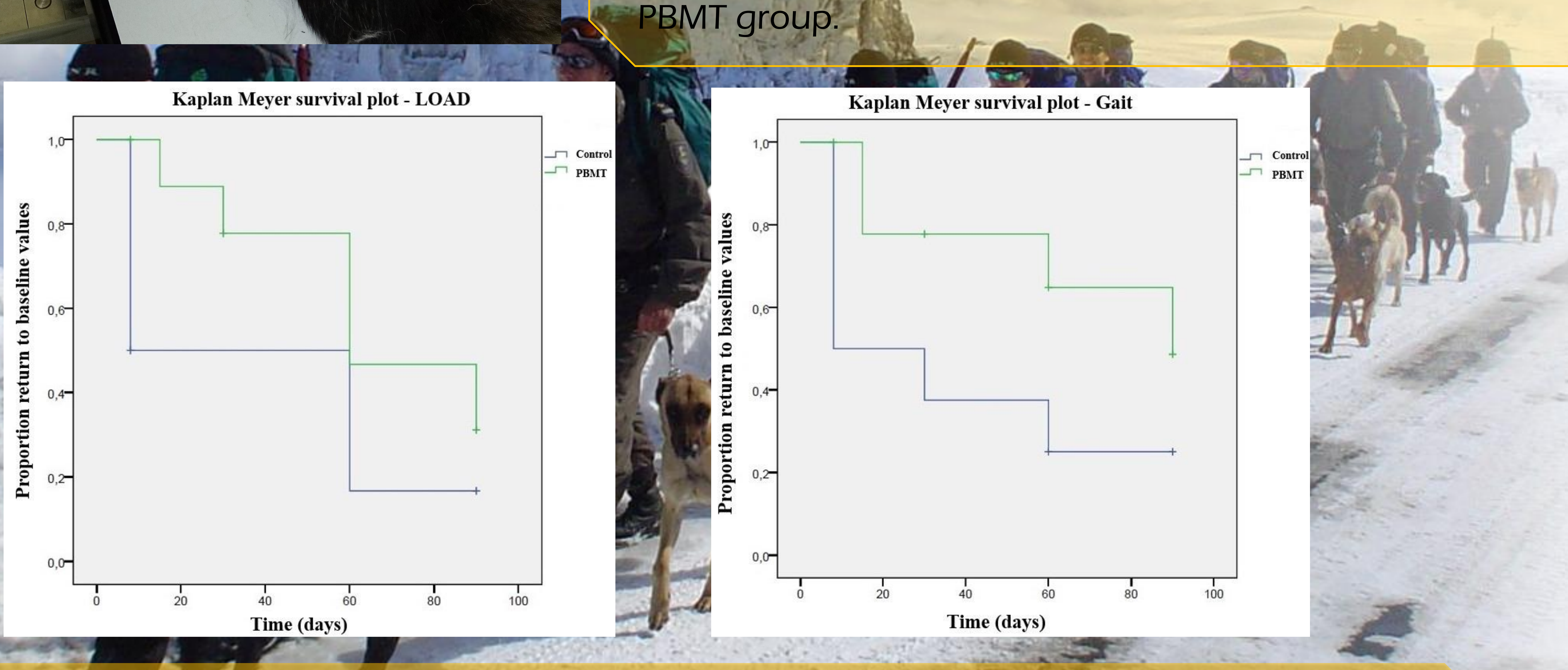

NPACG:

PBMT produced significant improvements in dogs with bilateral hip OA. 